\title{
Evaluation of Consumer Network Structure for Cosmetic Brands on Twitter
}

\author{
Yuzuki Kitajima ${ }^{1}$ \\ Graduate School of Science and \\ Engineering, Chuo University \\ Bunkyo-ku, Tokyo, Japan
}

\author{
Kohei Otake ${ }^{2}$ \\ School of Information and \\ Telecommunication Engineering \\ Tokai University, Minato-ku \\ Tokyo, Japan
}

\author{
Takashi Namatame ${ }^{3}$ \\ Faculty of Science and Engineering \\ Chuo University, Bunkyo-ku \\ Tokyo, Japan
}

\begin{abstract}
Since the early 2000s, the Internet has become increasingly popular for the development of information dissemination technology and as a platform for interaction. Therefore, the penetration rate of Social Networking Services (SNSs) is also increasing. Using the accounts created on SNSs, companies can disseminate information and communicate with users on SNSs for marketing purposes. Moreover, there are several influencer marketing activities that use influencers who are highly influential in their surroundings as marketing using SNSs. In this study, we aim to identify influencers on Twitter and consumer network structures for six cosmetic brands. Specifically, create a consumer network for each of the six cosmetic brands using follower data obtained from Twitter is created to identify the network structure. Furthermore, brand influencers were also identified. The consumer network of all six cosmetic brands was created to identify the influencers in the cosmetics industry. We compared the influencers of the brands with the influencers of the entire industry to examine any differences.
\end{abstract}

Keywords-Social networking services; community structure; network analysis; consumer network; influencer marketing

\section{INTRODUCTION}

With the development of information technology and the widespread use of Internet devices such as PCs and smartphones, the number of users on social networking services (SNSs) has been increasing yearly. SNSs are a form of media that allow users to easily share and disseminate information in real-time. They also allow two-way communication between the company and the consumer. In particular, the number of SNSs users in Japan is diverse in age groups and is expected to grow to approximately $80 \%$ of the population by 2020 [1]. According to a Japanese market research firm, LINE, Twitter, and Instagram are the most popular SNSs tools in Japan. LINE is a messaging tool that serves as a short message service (SMS). In contrast, Twitter and Instagram are SNSs tools that allow people with similar interests to communicate with each other anonymously and closely, and they are popular worldwide. In particular, Twitter ranks 15th in the global survey, while it ranks 2nd in Japan, which indicates a high penetration rate in Japan [2].

The spread of SNSs is not only limited to consumers, but also affects various companies. As shown in Fig. 1, number of corporate SNSs accounts has increased by approximately $10 \%$ from approximately 30\% in a year from 2017 to 2018 [3]. It is thought that the main reason for the increase in the number of corporate accounts on SNSs is that the diffusion of information by general users is expected to improve the product and brand recognition of companies. Considering the affinity between SNSs and marketing, we consider that marketing measures using SNSs are effective in industries with a large number of brands, such as the wholesale and retail industries. In addition, there are fashion and cosmetics industries where competition among brands is fierce, and it is assumed that these industries are also effective in improving brand recognition. Therefore, marketing using SNSs has become mainstream in recent years.

For example, Ferreira et al. [4] investigated the emergence of communities of co-commenters, that is, groups of users who often interact by commenting on the same posts and may be driving the ongoing online discussions. Their research used Instagram. The reason for this is that in recent years, social networking sites have been used as a source of information among young people, and politicians have also started to use this platform to spread information about political issues. In addition, Wang et al. [5] identified and tested the main factors related to SNS brand communities that can predict purchase intention. Their study suggested that companies should strategically manage consumers' SNS brand community experiences and commitment. Other theoretical implications and managerial implications were also discussed. This kind of research on customer behavior using social media is also conducted in the airline and hotel industries [6,7]. It is considered that the identification of influencers and consumer communities is an effective way to utilize SNSs in marketing. Influencers are people who have a large number of fans, especially SNSs, and have a large impact on the public. The identification of consumer communities is effective in identifying real customers of a brand.

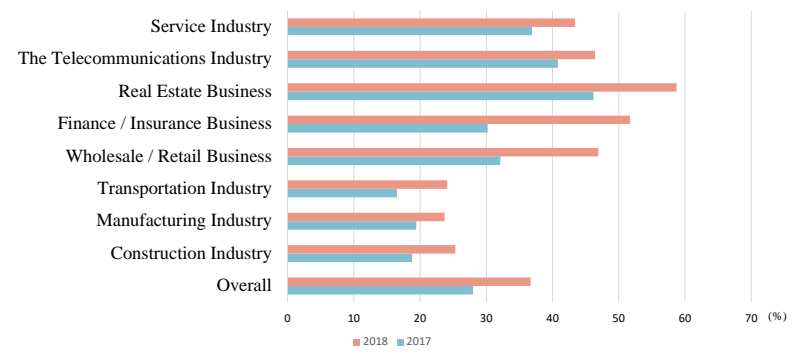

Fig. 1. Penetration of SNSs for each Industry. 
In previous research on network communities, Girvan et al. [8] focused on properties found in many networks, such as small-world and power-degree distributions. Specifically, they focused on detecting community structures where nodes are tightly connected and there are loose connections between them, which is the case in many networks. As a result, they proposed a method for finding community boundaries using a centrality index and detected useful community partitions in the validation data. Chunaev [9] conducted a survey of social network analysis. Specifically, they aimed to investigate and clarify the situation of community detection in social networks with node attributes. They found that community detection methods that deal with both network structure and attributes are effective for social network analysis. Mizuno et al. [10] classified CtoC (customer to customer) interactions by digitalization into four types and reviewed the research trends of each type. In their review, they suggested that the closer the consumers were to each other, the easier it was for information to propagate on SNSs. They also suggested that it is important to know which consumers or targets to reach out to first in the social graph. Himelboim et al. [11] created a network of users based on the conversations on Twitter and discussed the network structure by information flow. Through their research, they found that the network structure can be classified into six types. In addition, Pierri et al. [12] focused on the fact that malicious topics such as fake news are increasing in social networks. They compared two networks, one with correct information and the other with false information. As a result, they found that there was a difference in the structure between the two networks.

In contrast, as previous research on SNSs, Watanabe et al. [13] conducted a network analysis on Twitter for two international cosmetic retail brands. They focused on the brand's tweets and obtained the brand's tweets (tweets containing the @brand account name) and tweets containing the brand's hashtags (tweets containing the brand name). Then, they created an "ego network" representing the information propagation of official brand tweets and a "hashtag network" representing tweets containing brand hashtags to identify the way information propagates. As a result of their analysis, they found that brands could send out messages; however they could not restrict communication on the network and might not control the spread of information. Zhao et al. [14] used social network analysis to identify highly influential hashtags and hashtag communities to restructure the fashion industry using the development of Internet technology. Specifically, they extracted hashtags from text data on SNSs related to specific events and visualized them as a network to identify hashtags with high junctions. Wang et al. [15] conducted a study on whether marketing activities on SNSs are effective in improving market sales. Specifically, they used price, brand, and relationship customer equity to examine whether marketing activities on SNSs lead to customer purchase. As a result of their analysis, they found that SNSs marketing activities contribute to improving customer equity. They also found that customer equity contributes to customer loyalty and future sales. In addition, Miyake et al. [16] identified community of consumers in SNSs and analyzed their consumer network. In particular, they targeted five competing fashion brands in the fashion market and clarify the difference in community structure. Han et al. [17] focused on the influence of social media and created a network of the top $10 \mathrm{k}$ influencers on Twitter. They demonstrated how to surface influencers related to specific fashion topics that companies would be interested in. In their study, they used the follow, retweet (spread function), and mention (replies) functions all to create the network.

By using the structure of the consumer network, it is possible to identify the influencers in that brand. These brandspecific influencers are commonly referred to as microinfluencers and are attracting a lot of attention. However, identifying micro-influencers in a brand does not determine whether the micro-influencers are influential in the whole industry. We named these industry-wide influencers as "megainfluencers." To identify the mega-influencers, it necessary to clarify the network structure using consumers in the entire industry. We speculate that micro-influencers and megainfluencers are not necessarily the same. In addition, few related studies have identified both micro-influencers and mega-influencers. In particular, research on SNSs targeting fashion and cosmetic brands has focused on hashtags, but not so much on users. In this study, we focused on both microinfluencers and mega-influencers.

\section{PURPOSE}

The purpose of this study is to identify the consumer network structure of several cosmetic brands and to compare the differences in network structure among brands. In addition, it is compared whether there is a difference between micro-influencers and mega-influencers. The data of specific cosmetic brand accounts were collected from Twitter (one of the SNSs). Following, a consumer network for each brand and compared the consumer network structure for each brand was created, also identified the micro-influencers for each brand. Subsequently, the consumer networks of each brand were used to combine followers to create a consumer network for the entire cosmetics industry. Then, we identify and compare the mega-influencers that influence the entire industry.

\section{DAtaset AND DATA PROCESSING}

In this study, consumer networks were constructed using data from Twitter, one of the SNSs. We used the Twitter API to obtain data. Then, we processed the data to create a network.

\section{A. Hypothesis for Influencers on Twitter}

In this study, we defined the network structure of arbitrary followers on Twitter, as shown in Fig. 2. The area in Fig. 2 represents the number of users in the cosmetics industry. First, we define general consumers as Twitter users. Among these general consumers, micro-influencers influence each brand. Similarly, there are mega-influencers in the industry as a whole among general consumers. Based on this hypothesis, we extracted data from Twitter API.

\section{B. Dataset}

The targeted official accounts of the six cosmetic brands are in Table I. The year of establishment in Table I is the establishment of a cosmetic line. The following three criteria were used to select target brands: 
- Have a store in a department store.

- Have an official account on Twitter.

- Have more than 100,000 followers on Twitter.

The approximate number of followers and brand lineages of the six target brands are shown in Table II (as of January 8, 2022).

\section{Data Processing}

Next, we explain how to obtain data for each brand introduced in Section B. To clarify the network structure of consumers and influencers in this study, the following data are obtained as Fig. 3.

To explain the data structure, a brand A is picked up. First, we obtain a list of followers for a given brand. In Fig. 3, $f_{1}$ to $f_{n}$ represent the followers of brand A. Next, the list of users who follow the followers of brand A is obtained. In Fig. 3, the followers of brand A's follower $f_{1}$ are from $f f_{1}$ to $f f_{m}$. Then, we created a network using the data of the followers and users who follow the followers.

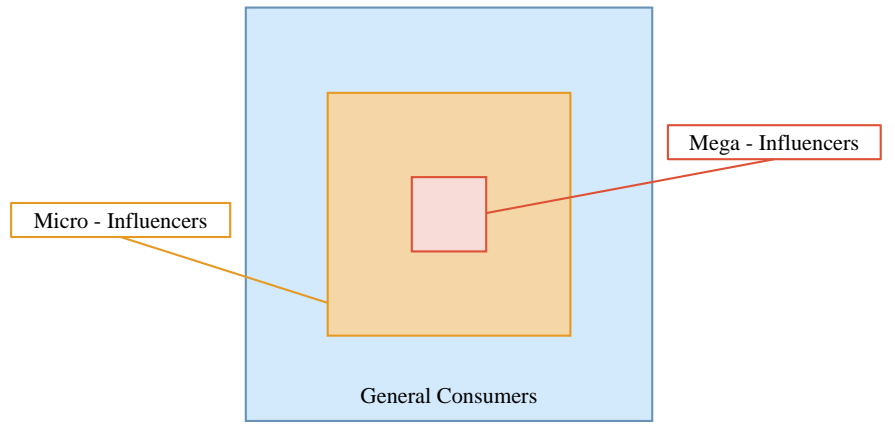

Fig. 2. Definition of Follower Hierarchy.

TABLE I. SUMmARY OF THE SIX BRANDS SELECTED

\begin{tabular}{|l|l|l|l|}
\hline Brand Names & Twitter ID & Trademark & Founded \\
\hline ADDICTION & @ StyleADDICTON & KOSE & 2009 \\
\hline Shu Uemura & @ shuuemurajp & L'Oreal & 1968 \\
\hline Paul \& Joe & @ PaulJoeBeauteJP & KOSE & 2002 \\
\hline ETVOS & @ etvos_jp & LVHM & 2007 \\
\hline CLINIQUE & @CliniqueJp & ESTEE LAUDER. & 1968 \\
\hline LANCOME & @Lancome_JP & L'Oreal. & 1935 \\
\hline
\end{tabular}

TABLE II. NUMBER OF FOLLOWERS OF THE TARGET BRANDS

\begin{tabular}{|l|l|l|l|}
\hline No. & Brand Names & Followers (10 thousand) & Fashion Line \\
\hline 1 & ADDICTION & 12.1 & Trendy \\
\hline 2 & Shu Uemura & 22.6 & Trendy \\
\hline 3 & Paul \& Joe & 11.4 & Cute \\
\hline 4 & ETVOS & 10.2 & Natural \\
\hline 5 & CLINIQUE & 11.5 & Natural \\
\hline 6 & LANCOME & 16.2 & Ellegance \\
\hline
\end{tabular}

In this study, we first created a follower network for each brand and identified the network structure. In addition, we identified influential micro-influencers and communities within the brand. Next, we created a network for the entire cosmetic brand. For the overall network, we used the nodes that were ultimately used in each brand's network. For the structure of the network, we used the Fruchterman-Reingold model [18] which is a dynamical model. It is also used PageRank [19] as a metric to identify the influencers. Modularity [20] was used to identify the user community. For node reduction, we used the magnitude of the edge weights described in Section 3B.

In this study, it is difficult to obtain all of the users who follow Brand A's followers, and it is impossible to obtain formation on accounts that are not public. Therefore, among are public accounts (blue box in Fig. 3). We used the first 1,000 accounts that were followed in a short period of time, ther than randomly selecting accounts. In addition, we "sweepstakes, winning" in the description from the scope of this study. To create a consumer network on Twitter, we used of the network among the acquired data. For the edges, we used users who followed the brand's followers (dotted boxes

Moreover, we weighted the edges in the following manner. As shown in Fig. 4, the followers of brand $\mathrm{A}$ are $f_{1}$ and $f_{2}$. followers of $f_{2}$ are $f f_{1}, f f_{3}, f f_{4} . f_{1}$ and $f_{2}$ have two common followers, $f f_{1}$ and $f f_{3}$. We used the number of common followers as the weights of the edges.

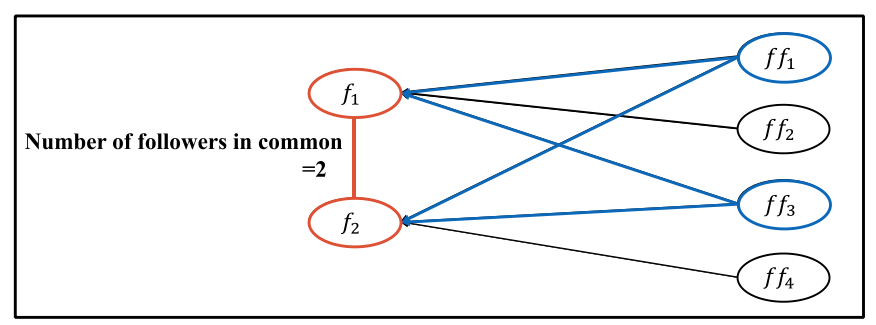

Fig. 4. Edge Weighting Method.

\section{VisUALIZATION OF FOLLOWER NETWORKS AND IDENTIFICATION OF MICRO-INFLUENCERS AND MEGA- INFLUENCERS}




\section{A. Follower Network Structure and Influencers of Each Cosmetic Brand}

In this study, we first visualized the follower network of each cosmetic brand. The number of nodes used is listed in Table III. To create the network, we used node reduction for accounts with more than one degree node. Edge weights were used to reduce the number of nodes. The number of nodes used in the network was set to approximately $30 \%$ of the initial number of nodes for each brand.

1) Network structure of "ADDICTION" and identifing of micro-influencers: Fig. 5 shows the visualization of the follower network of ADDICTION using all nodes with a degree greater than 1. From the degree distribution and Fig. 5, it was confirmed that the follower network of ADDICTION was scale-free. Fig. 6 shows a visualization of the addiction follower network after branch cutting. The color of the nodes indicates the community based on modularity. The size of the nodes is proportional to the size of the PageRank.

The average degree was 53.0. The number of communities detected by the modularity was three. For each community, Community 1 is brown, Community 2 is light blue, and Community 3 is beige. The density of the network was 0.226 . The top five users with the highest PageRank are listed in Table IV. The highest degree in the ADDICTION network was 142 and the lowest degree was 12. From Table IV, it was confirmed that micro-influencers with high PageRank in the ADDICTION network also tend to have high degree.

TABLE III. NUMBER OF NODES USED FOR EACH BRAND

\begin{tabular}{|l|l|l|l|}
\hline No. & Brand Names & $\begin{array}{l}\text { Initial Number of } \\
\text { Nodes }\end{array}$ & $\begin{array}{l}\text { Final Number of } \\
\text { Nodes }\end{array}$ \\
\hline 1 & ADDICTION & 767 & 236 \\
\hline 2 & Shu Uemura & 753 & 222 \\
\hline 3 & Paul \& Joe & 709 & 210 \\
\hline 4 & ETVOS & 761 & 229 \\
\hline 5 & CLINIQUE & 809 & 241 \\
\hline 6 & LANCOME & 791 & 237 \\
\hline
\end{tabular}

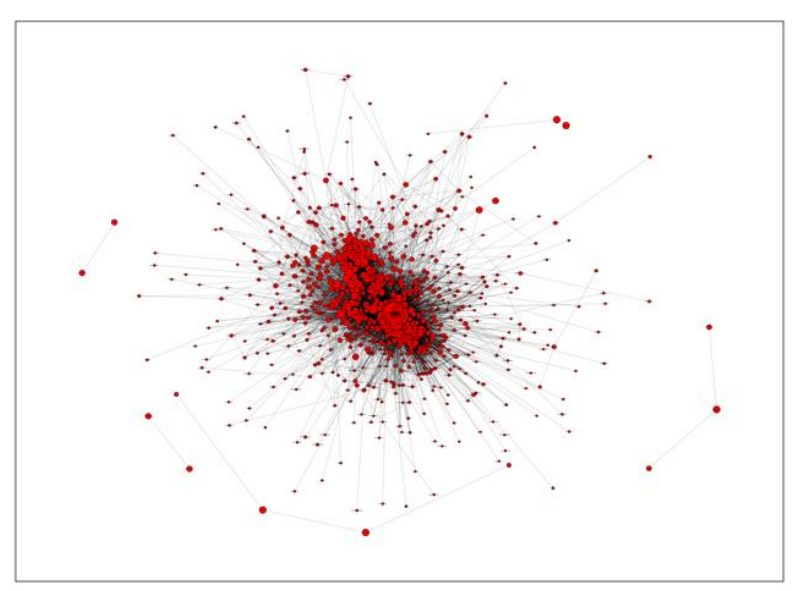

Fig. 5. Whole Consumer Network of ADDICTION.

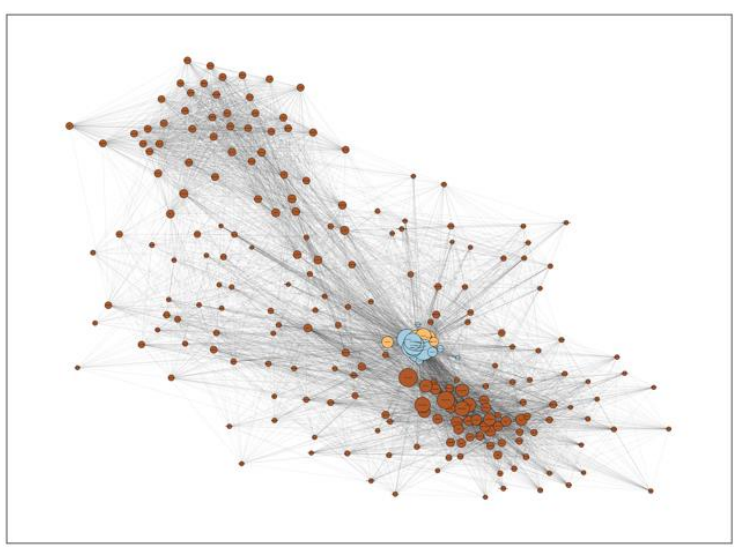

Fig. 6. ADDICTION Follower Network.

TABLE IV. TOP 5 USERS WITH High PAGERANK (ADDICTION)

\begin{tabular}{|l|l|l|l|l|}
\hline Rank & Users & PageRank & Degree & Community \\
\hline 1 & A & 0.0352 & 106 & 3 \\
\hline 2 & B & 0.0316 & 111 & 2 \\
\hline 3 & C & 0.0312 & 91 & 3 \\
\hline 4 & D & 0.0296 & 109 & 2 \\
\hline 5 & E & 0.0264 & 75 & 2 \\
\hline
\end{tabular}

Based on the users' tweeting tendencies, each community is characterized as, community 1 as a community of cosmetic lovers, community 2 as a community of interested in beauty and cosmetics, and community 3 as a daily tweet community. In addition, the density of communities 2 and 3 is higher than that of community 1 , and there is also a difference in the degree, so we determined that the network structure of the addiction is a "Centralized Network."

2) Network structure of "shu uemura" and identifing of micro-influencer: Fig. 7 shows the visualization of the follower network of Shu Uemura using all nodes with a degree greater than 1. From the degree distribution and Fig. 7, it was also confirmed that the follower network of Shu Uemura was scale-free.

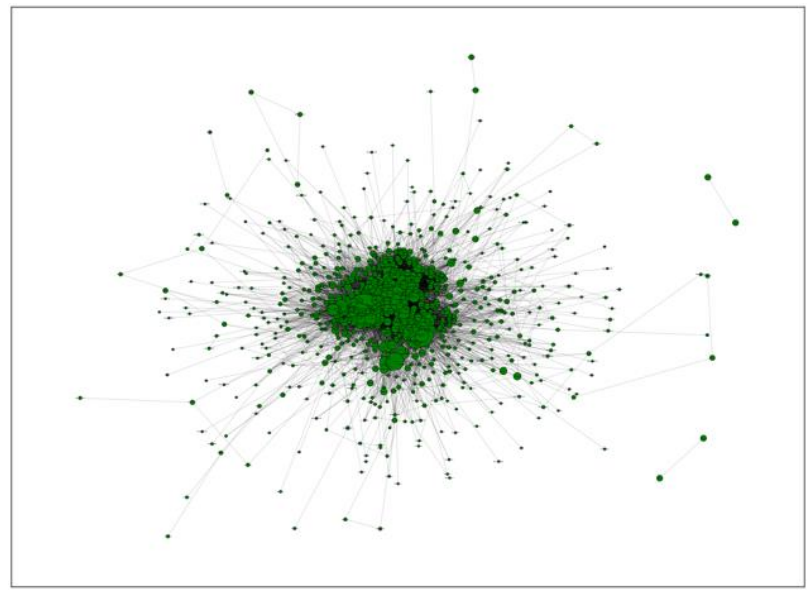

Fig. 7. Whole Consumer Network of Shu Uemura. 
Fig. 8 visualizes the Shu Uemura follower network after branch cutting. The average degree was 49.1 . The number of communities detected by the modularity was four. For each community, Community 1 is light purple, Community 2 is brown, Community 3 is pink, and Community 4 is light blue. The average network density was 0.222 .

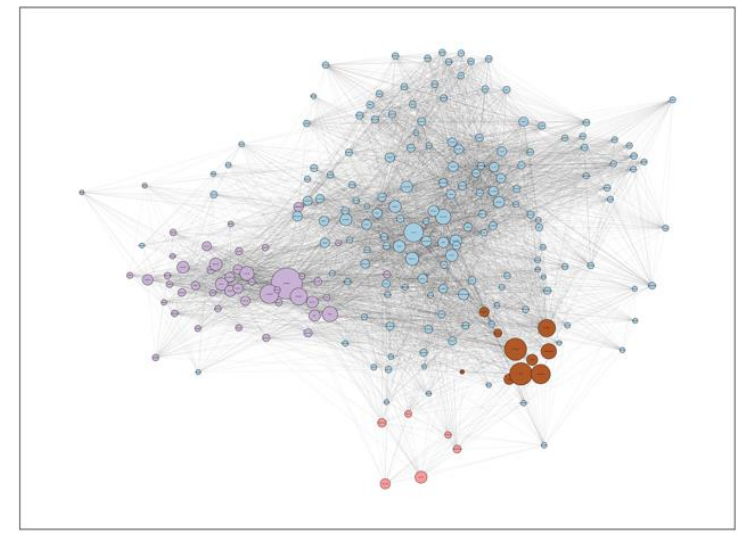

Fig. 8. Shu Uemura Follower Network.

TABLE V. Top 5 Users with High PageRank (Shu Uemura)

\begin{tabular}{|l|l|l|l|l|}
\hline Rank & Users & PageRank & Degree & Community \\
\hline 1 & F & 0.0563 & 120 & 1 \\
\hline 2 & G & 0.0299 & 49 & 2 \\
\hline 3 & H & 0.0288 & 101 & 2 \\
\hline 4 & I & 0.0223 & 22 & 2 \\
\hline 5 & J & 0.0216 & 118 & 4 \\
\hline
\end{tabular}

Table V lists the top five users with the highest PageRank. The highest degree in the Shu Uemura network was 132 and the lowest degree was 17. From Table V, it was confirmed that the degree of micro-influencers varied in the Shu Uemura network.

Based on the users' tweeting tendencies, we determined that community 1 is the cosplayer community (cosplayers are people who dress like anime and manga characters), community 2 is the homemaker community, community 3 is the community that likes cosmetics, and community 4 is the anime fan community. In addition, because the communities had many followers who were related to each other and the average degree was smaller than the others, we named them to be "Omnidirectional Network."

3) Network structure of "paul \& joe" and identifing of micro-influencers: Fig. 9 shows the visualization of the follower network of Paul \& Joe using all nodes with a degree greater than 1. From the degree distribution and Fig. 9, it was confirmed that the follower network of Paul \& Joe was scalefree. Fig. 10 shows a visualization of the Paul \& Joe follower network after branch cutting. The average degree was $48.9 \%$. The number of communities detected by the modularity was four. For each community, Community 1 is light purple, Community 2 is brown, Community 3 is light blue, and Community 4 is pink. The average network density was 0.234 .
Table VI lists the top five users with the highest PageRank. The highest degree in the Paul \& Joe network was 134 and the lowest degree was 14. From Table VI, it was confirmed that the Paul \& Joe network tends to have a high degree of micro-influencers, except for user $\mathrm{N}$.

Based on the users' tweet tendencies, we determined that community 1 was a daily tweet community, community 2 was the cosmetic lovers' community, community 3 was the color analysis community, and community 4 was communities derived from communities 1, 2, and 3. As shown in Fig. 7, communities 1 and 4 were formed by deriving from a larger community consisting of communities 2 and 3 . In addition, the average degree is not high. We declared Paul \& Joe network to be the "Centralized Network."

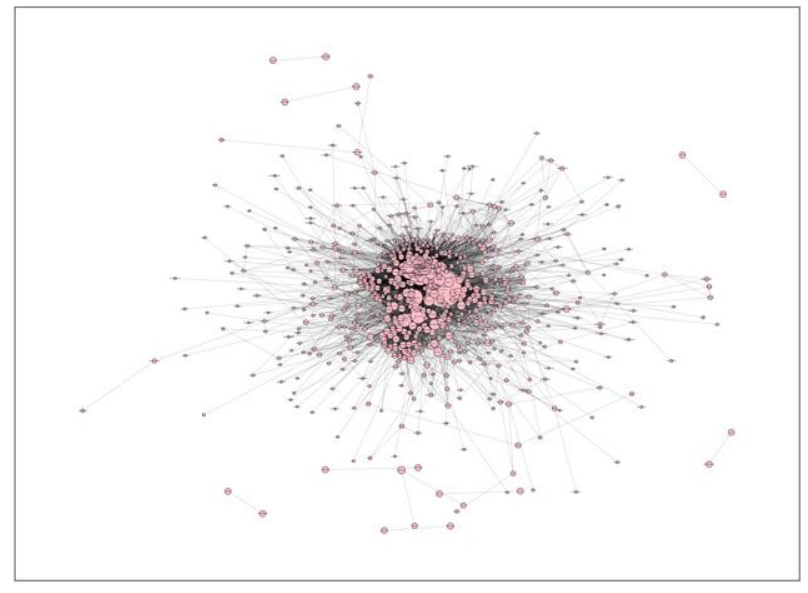

Fig. 9. Whole Consumer Network of Paul \& Joe.

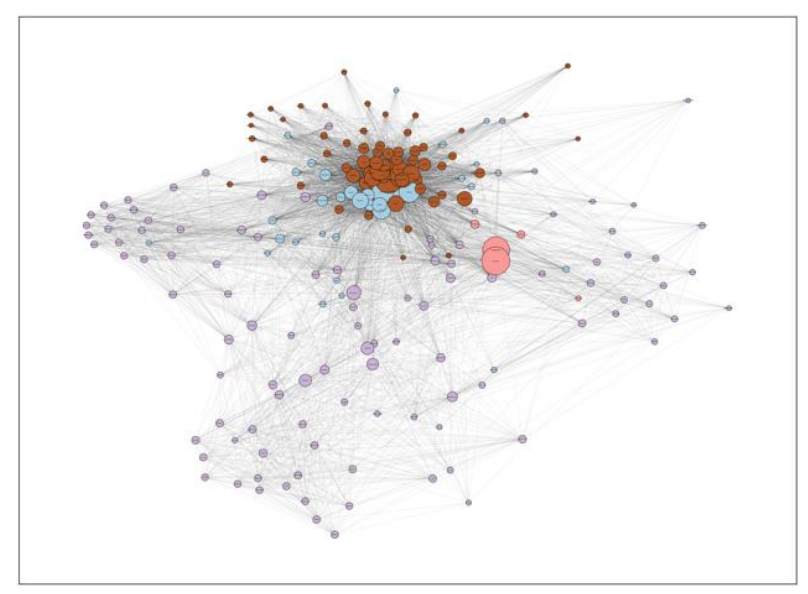

Fig. 10. Paul \& Joe Follower Network.

TABLE VI. TOP 5 USERS WITH High PAGERANK (PAUL \& JOE)

\begin{tabular}{|l|l|l|l|l|}
\hline Rank & Users & PageRank & Degree & Community \\
\hline 1 & K & 0.0390 & 106 & 4 \\
\hline 2 & L & 0.0387 & 126 & 2 \\
\hline 3 & M & 0.0366 & 119 & 2 \\
\hline 4 & N & 0.0353 & 54 & 4 \\
\hline 5 & O & 0.0290 & 105 & 2 \\
\hline
\end{tabular}


4) Network structure of "ETVOS" and identifing of micro-influencers: Fig. 11 shows the visualization of the follower network of the ETVOS using all nodes with a degree greater than 1. From the degree distribution and Fig. 11, it was confirmed that the follower network of the ETVOS was scalefree. Fig. 12 shows a visualization of the ETVOS follower network after branch cutting. The average degree was 51.1. The number of communities detected by the modularity was five. For each community, Community 1 is light purple, Community 2 is light blue, Community 3 is pink, Community 4 is brown, and Community 5 is brown. The average density of the ETVOS network is 0.224 .

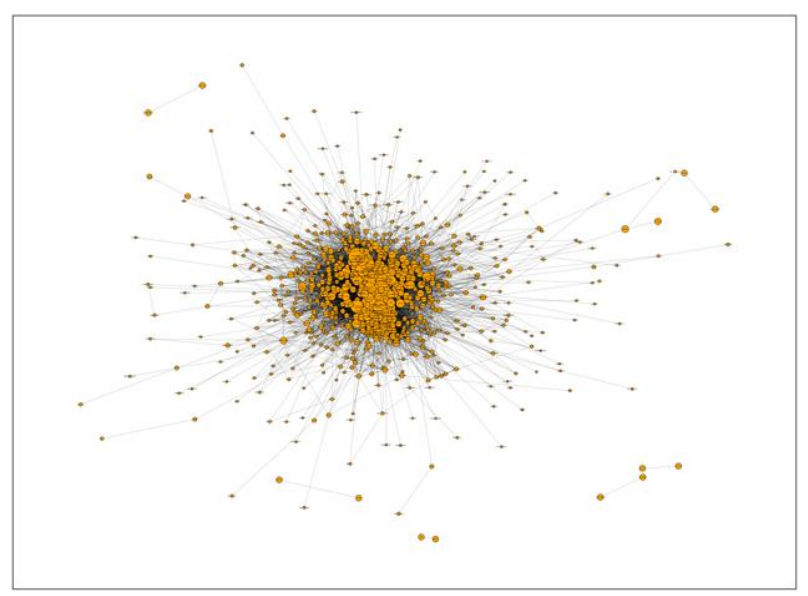

Fig. 11. Whole Consumer Network of ETVOS.

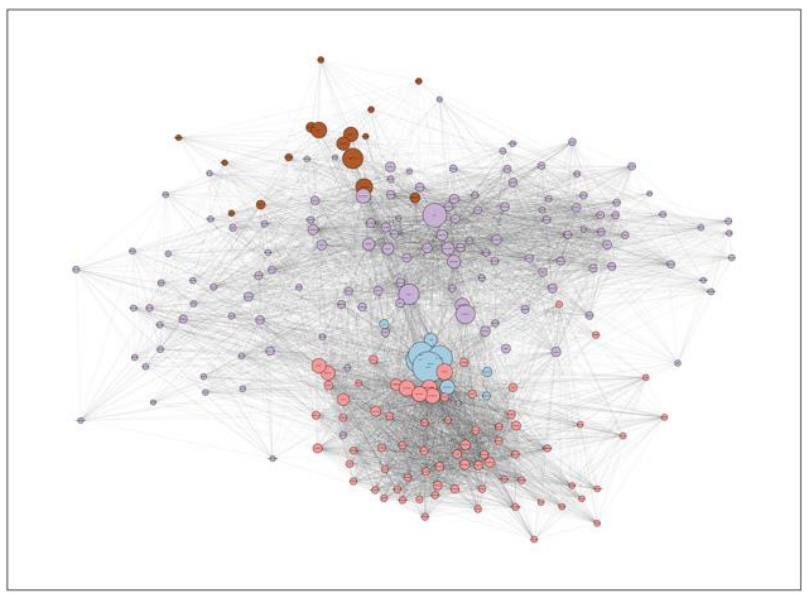

Fig. 12. ETVOS Follower Network.

TABLE VII. TOP 5 USERS WITH HIGH PAGERANK (ETVOS)

\begin{tabular}{|c|c|c|c|c|}
\hline Rank & Users & PageRank & Degree & Community \\
\hline 1 & P & 0.0459 & 115 & 2 \\
\hline 2 & Q & 0.0455 & 99 & 2 \\
\hline 3 & R & 0.0383 & 76 & 2 \\
\hline 4 & S & 0.0314 & 112 & 2 \\
\hline 5 & T & 0.0296 & 38 & 2 \\
\hline
\end{tabular}

Table VII lists the top five users with the highest PageRank. The highest degree in the ETVOS network was 170 and the lowest degree was 12. From Table VII, it was confirmed that the degree of micro-influencers varied in the ETVOS network. In particular, user $\mathrm{T}$ had a low degree but a high PageRank. In addition, we found that all the microinfluencers belonged to the same community. Based on users' tweet tendencies, community 1 was a daily tweet community, community 2 was a homemaker community, community 3 was an imprisoned community, community 4 was a cosmetic lovers' community, and community 5 was a lot of interest community. As the density of the ETVOS network tended to be low compared to the average degree of the ETVOS network, and as Fig. 8 shows, there was a connection between the communities. We named the network structure of ETVOS the "Omnidirectional Network."

5) Network structure of "CLINIQUE" and identifing of micro-influencers: Fig. 13 shows the visualization of the follower network of CLINIQUE using all nodes with a degree greater than 1 .

From the degree distribution and Fig. 13, the follower network of CLINIQUE was scale-free. Fig. 14 shows a visualization of the CLINIQUE follower network after branch cutting. The average degree was 68.3. The number of communities detected by the modularity was three. For each community, Community 1 is light blue, Community 2 is beige, and Community 3 is brown. The average density of the CLINIQUE network is 0.284 .

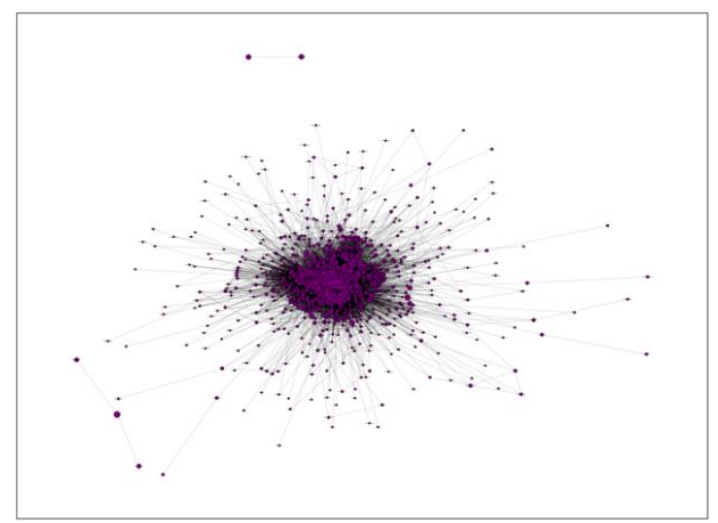

Fig. 13. Whole Consumer Network of CLINIQUE.

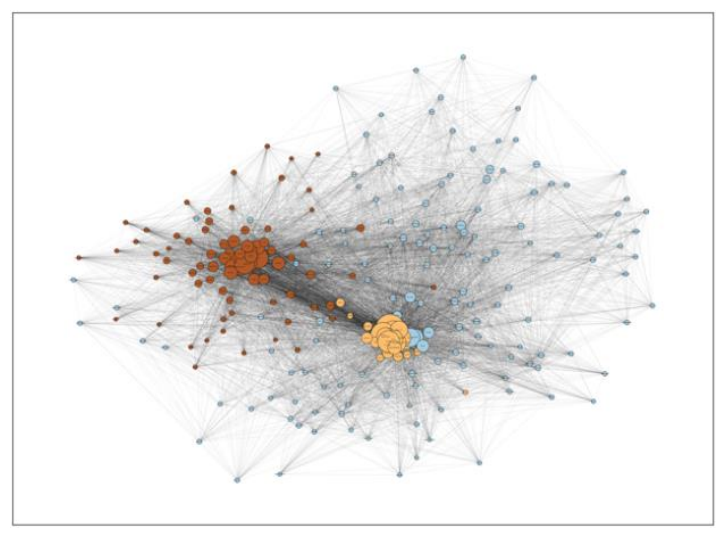

Fig. 14. CLINIQUE Follower Network. 
TABLE VIII. ToP 5 USERS WITH High PAGERANK (CLINIQUE)

\begin{tabular}{|l|l|l|l|l|}
\hline Rank & Users & PageRank & Degree & Community \\
\hline 1 & U & 0.0508 & 158 & 2 \\
\hline 2 & V & 0.0377 & 131 & 2 \\
\hline 3 & W & 0.0300 & 123 & 2 \\
\hline 4 & X & 0.0289 & 98 & 2 \\
\hline 5 & Y & 0.0254 & 126 & 3 \\
\hline
\end{tabular}

Table VIII lists the top five users with the highest PageRank. The highest degree in the CLINIQUE network was 185 and the lowest degree was 22. From Table VIII, it was confirmed that micro-influencers of the CLINIQUE network tended to be high degree, although their PageRank varied. Based on users' tweet tendencies, we determined that community 1 had many hobbies community, community 2 was a homemaker community, and community 3 was a cosmetic lovers' community. From Fig. 9, it seems that communities 1,2 , and 3 are divided into two groups. We determined that the connections between users in these two communities were high, based on the average degree of the network. Therefore, we named CLINIQUE network to be "Dual Network."

6) Network structure of "LANCOME" and identifing of micro-influencers: Fig. 15 shows the visualization of the follower network of the LANCOME using all nodes with a degree greater than 1. From the degree distribution and Fig. 15, the follower network of the LANCOME was scalefree. Fig. 16 shows a visualization of the LANCOME follower network after branch cutting. The average degree was 58.0. The number of communities detected by the modularity was four. For each community, Community 1 is brown, Community 2 is pink, Community 3 is light blue, and Community 4 is light purple. The average density of the LANCOME network is 0.246 .

Table IX lists the top five users with the highest PageRank. The highest degree in the LANCOME network was 165 and the lowest degree was 22. From Table IX, it was confirmed that the degree was proportionally high to the PageRank of micro-influencers in the LANCOME network. Based on users' tweet tendencies, we determined community 1 as a cosmetic lovers' community, community 2 had a lot of hobbies community, community 3 was a cosmetic lovers and sweepstakes community, and community 4 was a talent lovers' community. From Fig. 16, we found that there are two large communities, one by Community 1, 2, and 3 and the other by Community 4.

Although the average degree of the network is not high, there are connections between the two large communities. Therefore, we named the network structure of LANCOME as a "Dual Network."

\section{B. Visualization of Follower Networks across the Cosmetics Industry and Identify Mega-influencers}

Next, in order to find mega-influencers, we created a follower network for all six brands using the follower users used in Section A. We reduced the number of nodes by using the edge weights calculated in Section 3-B. We used 357 nodes for the final visualization of the 1,277 nodes remaining in Section A. Table X shows the breakdown of the number of nodes for each brand used in the final network visualization.

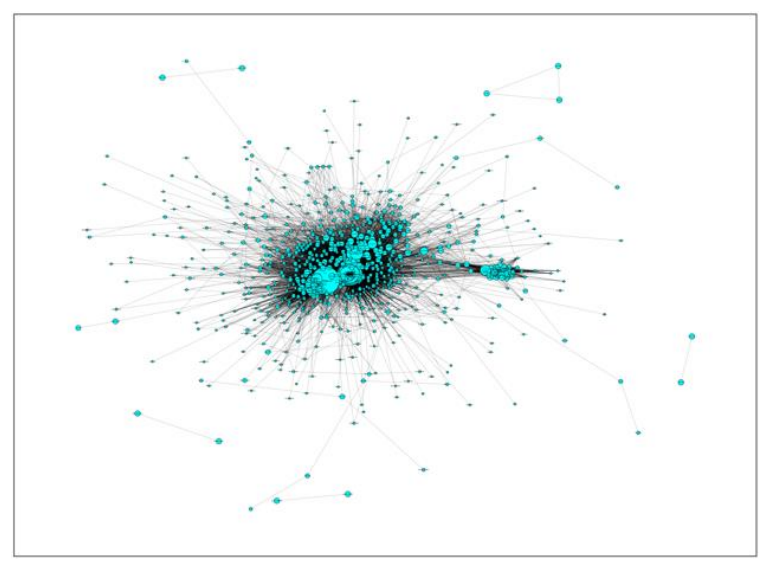

Fig. 15. Whole Consumer Network of LANCOME

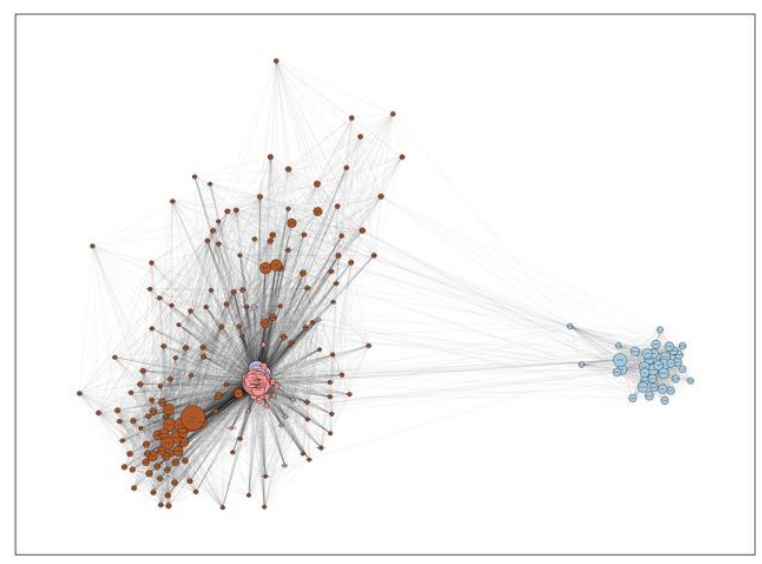

Fig. 16. LANCOME Follower Network.

TABLE IX. TOP 5 USERS WITH High PAgERANK (LANCOME)

\begin{tabular}{|l|l|l|l|l|}
\hline Rank & Users & PageRank & Degree & Community \\
\hline 1 & Z & 0.0330 & 125 & 1 \\
\hline 2 & AA & 0.0265 & 115 & 2 \\
\hline 3 & AB & 0.0234 & 165 & 2 \\
\hline 4 & AC & 0.0232 & 101 & 1 \\
\hline 5 & AD & 0.0209 & 132 & 2 \\
\hline
\end{tabular}

TABLE X. NODE COLOR AND NUMBER OF NODES FOR EACH BRAND

\begin{tabular}{|l|l|l|}
\hline Brand Names & Colors & Number of Nodes \\
\hline ADDICTION & Pink & 47 \\
\hline Shu Uemura & Yellow & 39 \\
\hline Paul \& Joe & Light green & 42 \\
\hline ETVOS & Light blue & 36 \\
\hline CLINIQUE & Light purple & 58 \\
\hline LANCOME & Orange & 106 \\
\hline Multiple & Brown & 29 \\
\hline
\end{tabular}


Fig. 17 shows the results of visualizing the network using the nodes listed in Table X. Some users followed more than one brand in Fig. 17 (29 nodes as shown in Table X). The network average degree was 47.3.

As a result of using modularity for the entire brand network, we detected five communities (Fig. 18). Community 1, light blue; Community 2, light green; Community 3, pink; Community 4, orange; Community 5, purple; and Community 6, brown. From Fig. 17 and 18, we found that the upper-right community (community 6) follows LANCOME. In addition, we found that the lower left community (community 3) followed the Shu Uemura. So, there are communities in the whole network where only the followers of a particular brand stick together.

Table XI lists the top ten users with the highest PageRank. "Brand" in Table XI represents the brands that each user is following. Comparing Table XI with the micro-influencers for each brand, it was confirmed that half of the mega-influencers were not included in the micro-influencers for each brand. Comparing the percentage of communities, we found that the percentage of communities in Community 1 (the orange community in Fig. 18) was high, indicating that it was the central community in the overall network.

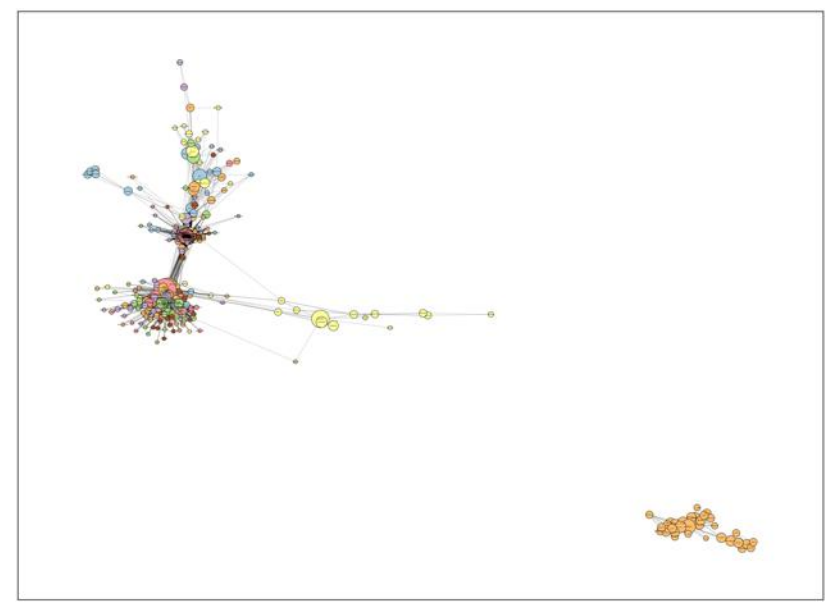

Fig. 17. Network of Coloration by Brand.

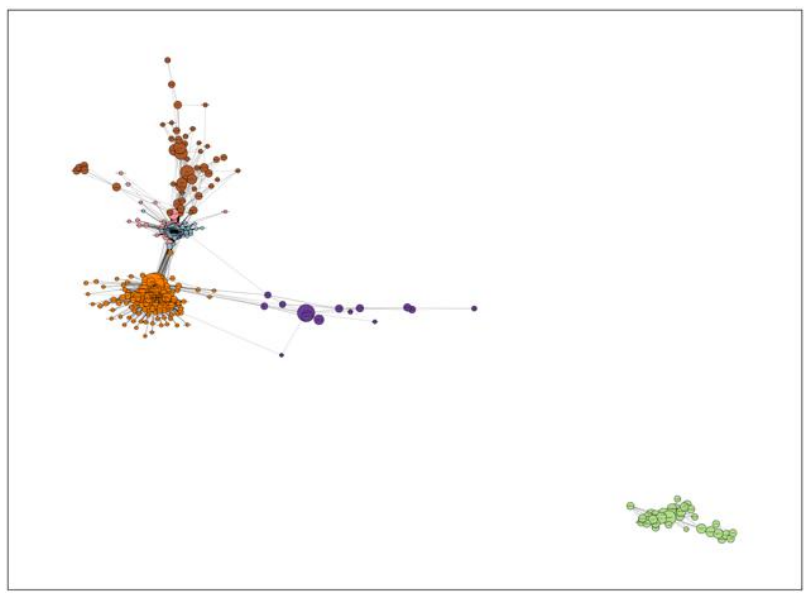

Fig. 18. Network Diagram of the Entire Brand.
TABLE XI. TOP 10 USERS WITH HIGH PAGERANK

\begin{tabular}{|l|l|l|l|l|}
\hline Rank & Users & PageRank & Community & Brand \\
\hline 1 & Z & 0.0227 & 1 & LANCOME \\
\hline 2 & AE & 0.0177 & 1 & ADDICTION \\
\hline 3 & AF & 0.0129 & 1 & CLINIQUE \\
\hline 4 & Y & 0.0125 & 1 & CLINIQUE \\
\hline 5 & AG & 0.0114 & 1 & ETVOS \\
\hline 6 & F & 0.0107 & 3 & Shu Uemura \\
\hline 7 & AH & 0.0104 & 1 & LANCOME \\
\hline 8 & U & 0.0104 & 5 & CLINIQUE \\
\hline 9 & AI & 0.0102 & 1 & CLINIQUE \\
\hline 10 & A & 0.0090 & 2 & ADDICTION \\
\hline
\end{tabular}

\section{DISCUSSION}

First, it is confirmed that all brand consumer networks were scale-free networks, as shown in Fig. 5, 7, 9, 11, 13, and 15. Therefore, we considered that some micro-influencers mainly spread the information to other users who follow each micro-influencer.

Fig. 19 summarizes the results of classifying the follower network of the six brands into three network structures. The "Omnidirectional Network" refers to a network in which the entire community is universally connected. The "Dual Network" is a network in which the entire network is divided into two major communities. In these network structures, it is obtained those characteristics similar to those of the smallworld in the degree distribution after node reduction. The "Centralized Network" is a network in which nodes are distributed to derive from one central community. From Tables V and VII, we confirmed that the micro-influencers of Shu Uemura and ETVOS, which are "Omnidirectional Network," varied degree. On the other hand, we confirmed that degrees of the micro-influencers of the four brands with "Dual Network" and "Centralized Network" tended to be high in proportion to PageRank from Tables IV, VI, VIII, and IX. In the omnidirectional network, there are various communities, and the communities are all related with each other. For this reason, we speculated that information tends to be transmitted easily even if the degree is not high, and PageRank may become high. On the other hand, "Dual Network" and "Centralized Network" are locally divided into one or two communities. Therefore, we considered that the value of PageRank was proportional to the value of degree.

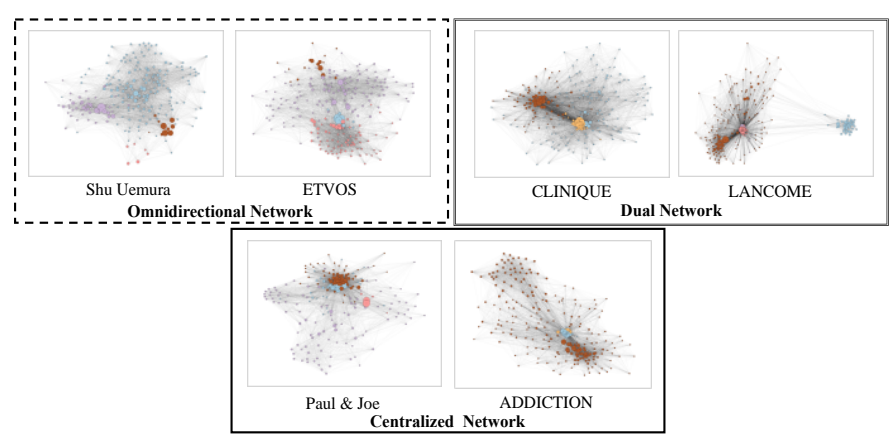

Fig. 19. Classifying Network Structures. 
From the results of this classification and the comparison of the brand lineages in Table II, we inferred that the brand lines were not proportional to the network structure. As for the communities of each brand, it is observed a community of tweets about sweepstake entries in all networks, which was not directly related to cosmetics. These communities were unique to Twitter and considered the effect of a campaign to increase followers and product recognition. As a characteristic community, Shu Uemura had a community that liked animation and comic, and LANCOME had a community that liked celebrities. Shu Uemura sells cosmetics with tie-ups with manga and anime characters every winter, and LANCOME has Japanese celebrities as ambassadors, actively appearing in commercials and advertisements. Therefore, we consider that there is a good possibility that users, who prefer the tie-up side, but not the cosmetics themselves, are interested in the products and become fans. In contrast, Paul \& Joe and ADDICTION specialize in communities of users who are interested in cosmetics and beauty. Paul \& Joe's cosmetics with cat motifs are popular, and ADDICTION's single eyeshadow colors are abundant and popular. Therefore, we considered that they might be more likely to attract collectors than other brands.

Based on the tweeting tendencies of users belonging to each community, we roughly classified them into communities with similarities by community detection. However, some of the derived communities were inconsistent in their tweet content and self-introductions. It is assumed that this was due to the modularity of the community detection.

Table XII summarizes the results of the micro-influencers for each brand. As shown in Fig. 17, some users followed more than one brand. However, Table XII shows that there were no overlapping users among the six brands. Therefore, we inferred that user who are influential on one brand do not necessarily influence other brands. In addition, comparing Tables XI and XII, which show the mega-influencers, only five users (A, F, U, Y, and Z) were included in both tables. Therefore, we inferred mega-influencers were not necessarily micro-influencers for each brand. In contrast, Table XI shows that all mega-influencers followed only one brand. Given these facts, we considered whether a person following multiple brands was not a criterion for being a megainfluencer.

TABLE XII. TOP 5 MICRO-INFLUENCERS FOR EACH BRAND

\begin{tabular}{|l|l|l|l|l|l|l|}
\hline & $\begin{array}{l}\text { ADDICT } \\
\text { ION }\end{array}$ & $\begin{array}{l}\text { Shu } \\
\text { Uemu } \\
\text { ra }\end{array}$ & $\begin{array}{l}\text { Paul\&J } \\
\text { oe }\end{array}$ & $\begin{array}{l}\text { ETVO } \\
\text { S }\end{array}$ & $\begin{array}{l}\text { CLINIQ } \\
\text { UE }\end{array}$ & $\begin{array}{l}\text { LANCO } \\
\text { ME }\end{array}$ \\
\hline 1 & A & F & K & P & U & Z \\
\hline 2 & B & G & L & Q & V & AA \\
\hline 3 & C & H & M & R & W & AB \\
\hline 4 & D & I & N & S & X & AC \\
\hline 5 & E & J & O & T & Y & AD \\
\hline
\end{tabular}

Furthermore, none of the users following Paul \& Joe are included in Table XI. From the node breakdown in Table X, the number of nodes was not extremely low, and the density of brand consumer networks was not extremely small. One of the reasons for this was that the users who followed Paul \& Joe were biased toward accounts that specialized in cosmetics. Contrarily, although Shu Uemura had the smallest number of nodes in the overall network, users who followed Shu Uemura were included in the top ten mega-influencers. Considering the fact that Shu Uemura's followers come from various lines of users, we considered that even in the cosmetics industry, users who were connected to various lines of users were more likely to become mega influencers who influence the whole network. However, as shown in section 4-B, even in the entire brand network, there were communities that consisted only of followers of a particular brand. We thought that users who belonged to such communities were not necessarily influential to the entire industry. Therefore, to identify mega-influencers, we considered it necessary to take into account their community affiliation even if their PageRank was high.

\section{CONCLUSION AND FUTURE WORK}

In this study, we identified and compared the consumer network structures of six cosmetic brands. We also identified and compare the micro-influencers for each brand with the mega-influencers for the brands as a whole. We used network analysis. The consumer network was visualized using the Fruchterman-Reingold model, PageRank, and modularity to reveal the community.

As a result of the analysis, we classified the six cosmetic brands into three network structures. We named the three networks "Omnidirectional Network," "Centralized Network," and "Dual Network." It was also found that the microinfluencers with high PageRank and high degree for each brand were different. After visualizing the entire network, we classified the network into six communities. Of the six brands, only Paul \& Joe's followers were not included in the top 10 mega-influencers with high PageRank. Comparing microinfluencers and mega-influencers, it was found that microinfluencers are not always mega-influencers.

In future work, we believe that a generalization of consumer networks in the cosmetics industry will be possible by conducting the same analysis for cosmetic brands that match the same conditions as in this study and comparing the results. In particular, the recent outbreak of COVID-19 has led to a decline in sales in the cosmetics industry in Japan, so we consider this to be an effective marketing strategy. In addition, although we used modularity for community detection in this study, some communities could not be judged well based on users' tweeting tendencies. Therefore, we believe that network analysis using metrics other than modularity will allow us to evaluate networks from different perspectives.

\section{ACKNOWLEDGMENT}

This work was supported by JSPS KAKENHI Grant Number 19K01945, $21 \mathrm{H} 04600$ and 21K13385.

\section{REFERENCES}

[1] ICT Research \& Consulting Inc., Survey on SNSs Usage Trends in 2020 , https://ictr.co.jp/report/20200729.html/, last viewed on Jan. 29, 2022.

[2] Statista, Most Popular Social Networks Worldwide as of October 2021, Ranked by Number of Active Users, https:/www.statista.com/statistics/ 272014/global-social-networks-ranked-by-number-of-users/, last viewed on Jan. 20, 2022. 
[3] Ministry of Internal Affairs and Communications of Japan, Results of Telecommunications Usage Trends Survey in 2018, https://www.soumu. go.jp/johotsusintokei/statistics/data/190531_1.pdf, last viewed on Jan. 29, 2022.

[4] Carlos H.G. Ferreira, F. Murai, Ana P. C. Silva, J. M. Almeida, M. Trevisan, L. Vassio, M. Mellia and I. Drago, "On the Dynamics of Political Discussions on Instagram: A Network Perspective," Online Social Networks and Media, Vol. 25, 2021.

[5] X. W. Wang, Y. M. Cao, C. Park, "The Relationships Among Community Experience, Community Commitment, Brand Attitude, and Purchase Intention in Social Media," International Journal of Information Management, Vol. 49, pp. 475-488, 2019.

[6] R. Huang, S. Ha, S. H. Kim, "Narrative Persuasion in Social Media: An Empirical Study of Luxury Brand Advertising," Journal of Research in Interactive Marketing, Vol. 12, pp. 274-292, 2018.

[7] E. J. Seo, J. W. Park, "A Study on the Effects of Social Media Marketing Activities on Brand Equity and Customer Response in the Airline Industry," Journal of Air Transport Management, Vol. 66, pp. 36-41, 2018.

[8] M. Girvan, M. E. J. Newman , "Community Structure in Social and Biological Networks," Proceedings of the National Academy of Sciences, Vol. 99 (12), pp.7821-7826, 2002.

[9] P. Chunaev, "Community Detection in Node-attributed Social Networks: A Survey," Computer Science Review, Vol. 37, 2020.

[10] M. Mizuno, H. Onishi, S. Shibuya, H. Yamamoto, "C2C Interactions in the Digital Media Environment : A Review and Perspective," Journal of Marketing Science, Vol. 26, pp. 7-39, 2018 (Japanese).

[11] I. Himelboim, M. A. Smith, L. Rainie, B. Shneiderman and C. Espina, "Classifying Twitter Topic-Networks Uning Social Network Analysis," Social Media + Society, Vol. 3, 2017.
[12] F. Pierri, C. Piccardi, S. Ceri, "Topology Comparison of Twitter Diffusion Networks Effectively Reveals Misleading Information," Scientific Reports, Vol. 10, 2020.

[13] N. M. Watanabe, J. Kim, J. Park, "Social Network Analysis and Domestic and International Retailers: An Investigation of Social Media Networks of Cosmetic Brands," Journal of Retailing and Consumer Services, Vol.58, 2021.

[14] L. Zhao, C. Min, "The Rise of Fashion Informatics: A Case of DataMining-Based Social Network Analysis in Fashion," Clothing and Textiles Research Journal, Vol. 37 (2), pp. 1-16, 2019.

[15] H. Wang, E. Ko, A. Woodside, J. Yu, "SNS Marketing Activities as a Sustainable Competitive Advantage and Traditional Market Equity," Journal of Business Research, Vol. 130, pp373-383, 2021.

[16] S. Miyake, K. Otake, T. Namatame, "Analysis of Consumer Community Structure within Social Media -A Case Study of Competing Brands in Japanese Fashion Market-," International Academy of Business and Economics, Vol. 19, pp. 65-80, 2019.

[17] J. Han, Q. Chen, X. Jin, W. Xu, W. Yang, S. Kumar, L. Zhao, H. Sundaram and R. Kumar, "FITNet: Identifying Fashion Influencers on Twitter," Proceedings of the ACM on Human-Computer Interaction, Vol. 5, pp. 1-20, 2021.

[18] T. M. J. Fruchterman, E. M. Reingold, "Graph by Force-directed Placement," Software Practice and Experience, Vol. 21 (11), pp. 11291164, 1991.

[19] L. Page, S. Brin, R. Motwani, T. Winograd, "The PageRank Citation Ranking: Bring Order to the Web," Technical Report of the Stanford Digital Library Technologies Project, 1998.

[20] M. E. J. Newman,"Modularity and Community Structure in the Networks," Proceedings of the National Academy of Science of the United States of America, Vol. 103, pp. 8577-8582, 2006. 